\title{
Detrusor muscle in the initial TURBT specimen and recurrence rate at first check cystoscopy in non-muscle invasive bladder cancer
}

\author{
Serozsha Goonewardena ${ }^{1}$, Chandu de Silva ${ }^{2}$, Umesh Jayarajah ${ }^{1}$, Viraj de Silva ${ }^{1}$, Balasubramaniam \\ Sathesan ${ }^{1}$, Prabath Pathirathne ${ }^{1}$, Kathirgamathamby Vickneswaran ${ }^{1}$, Manoj Hilary Fernando ${ }^{1}$ \\ ${ }^{1}$ Department of Urology, National Hospital of Sri Lanka, Colombo, Sri Lanka \\ ${ }^{2}$ Department of Pathology, Faculty of Medicine, University of Colombo, Sri Lanka
}

Keywords: Detrusor muscle; transurethral resection of bladder tumour; recurrence rate; non-muscle invasive bladder cancer

\begin{abstract}
\section{Introduction}

The presence of detrusor muscle (DM) is an essential component in "complete" transurethral resection of bladder tumour (TURBT) specimen. This study analyses patients who were subjected to a "complete" first TURBT to determine the presence/absence of DM in the specimen.
\end{abstract}

\section{Methodology}

Newly diagnosed non-muscle invasive bladder cancer (NMIBC) from 1-April-2007 to 31-March-2017(10-years) were retrospectively analysed at National Hospital of Sri Lanka. All TURBTs performed at initial diagnosis were analysed to determine the presence of DM in the specimen, the recurrence rate at first check cystoscopy (FCC) at 3 months and the association with surgeon's experience.

\section{Results}

Of 181 TURBT, 99(54.7\%) were done by Consultant Urological Surgeon (CUS) and $82(45.3 \%)$ by senior registrars (SR). The overall DM positivity rate was $59.7 \%(\mathrm{n}=$ 108/181), for CUS: $63.6 \%(n=63 / 99)$ and SRs $54.9 \%$ $(n=45 / 82)(p=0.232)$; for pTa tumours:-CUS: 46.5\%vs.SRs:50 \%( $\mathrm{p}=0.751)$ and for $\mathrm{pT} 1$ tumours:-CUS: $76.8 \%$ vs.SRs: $59.5 \%(p=0.067)$. Analysis of those who were followed up $(145 / 181,80.1 \%)$ showed a positive recurrence at FCC in $27.1 \%(23 / 85)$ and $30.0 \%(18 / 60)$ when DM was present and absent in the first TURBT specimen respectively $(\mathrm{OR}=0.87(95 \%$-CI: 0.41-1.8, $\mathrm{p}=0.7)$.

\section{Conclusion}

Clinically, DM positivity rate has prognostic significance only in the pT1 category of NMIBC. For pT1 tumours, the positive DM rates for the CUS and SRs were $76.8 \%$ and $59.5 \%$ respectively. The indifference in the recurrence rate

\footnotetext{
Correspondence: Serozsha Goonewardena

E-mail: drsasgurol@yahoo.com

Received: 23-12-2019 Accepted: 23-04-2020

(i) https://orcid.org/0000-0002-B53-3356

DOI: http://doi.org/10.4038/sljs.v38i1.8653
}

may be due to the considerable proportion of lost to follow up in our study. Nevertheless, measures should be taken to enhance the DM positivity rates among urologists to achieve accurate staging and better prognosis.

\section{Introduction}

Bladder malignancies are the fourth commonest male cancers worldwide[1]. Furthermore, bladder cancer is common among Sri Lankan males and also seen among females [2,3]. Transurethral resection of bladder tumour (TURBT) is currently the standard of care for the less aggressive papillary bladder cancer with subsequent intravesical mitomycin within 6 hours [4,5]. A complete TURBT in non-muscle invasive bladder cancer (NMIBC) should encompass (i) resection of all visible tumour(s) (ii) resection of apparently normal mucosa $(\sim 1 \mathrm{~cm})$ on the border of the tumour, and (iii) resection of part of the detrusor muscle (muscularis propria) at the tumour base [6].

The uropathologist should state the stages as pTx in the absence of detrusor muscle (DM) in the TURBT specimen, particularly when there is presence of tumour in the lamina propria (i.e. pT1 or higher), emphasising the requirement to perform a restaging TURBT. NMIBC can quite confidently be reported only when DM present in the specimen is uninvolved by urothelial bladder cancer.

The present study assesses the patients with bladder cancer who underwent a "complete" first TURBT to describe the occurrence of DM in the resection specimen, to predict the risk of tumour recurrence at first check cystoscopy (usually 3 months later) and whether these are dependent on surgeon's experience.

\section{Materials and methods}

A retrospective analysis was carried out of newly diagnosed urothelial bladder cancer patients with a final diagnosis of NMIBC from 1 April 2007 to 31 March 2017 in a single urology unit at National Hospital of Sri Lanka. Ethical approval was received from the Ethical review committee of the National Hospital of Sri Lanka (No:AAJ/ETH/COM/2017-13). All TURBTs done at the initial diagnosis by the consultant urological surgeon (CUS) (with more than 15 years of experience) and four consecutive senior registrars (SR) (each 
Table 1. Presence of detrusor muscle in the TURBT specimen in small and large bladder tumours dependent on 5 surgeons studied

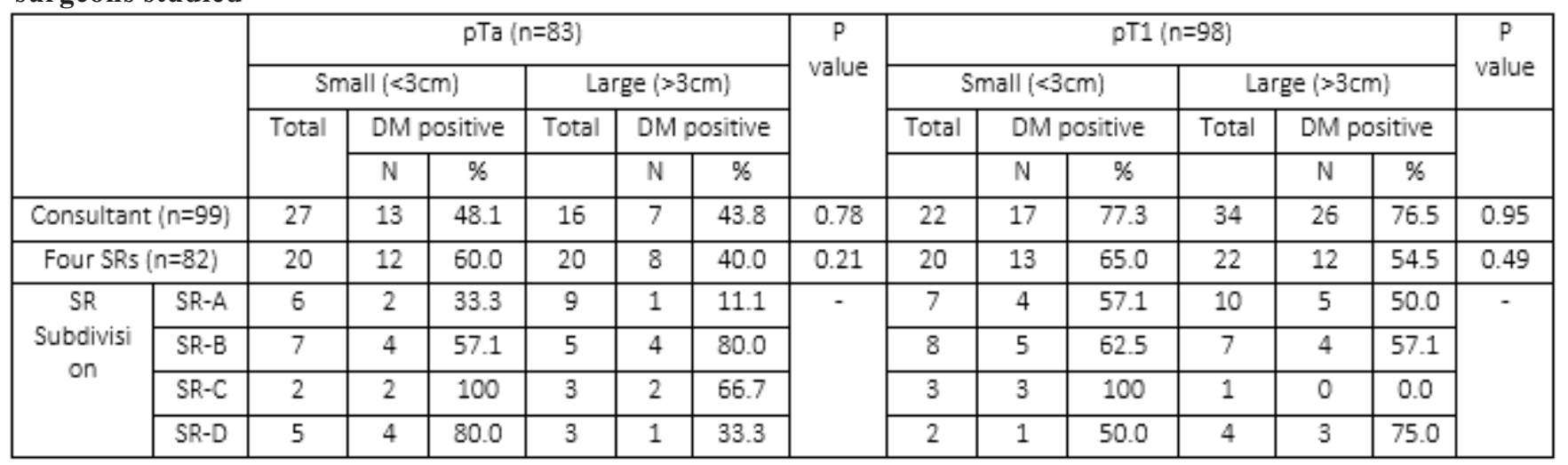

with a minimum 6 months training) were analysed to determine the presence of DM in the specimen and the recurrence rate at first check cystoscopy at 3 months. None of the patients received an intravesical mitomycin after TURBT.

The specimen were analysed and the presence of DM was looked at for gender and focality (for all surgeons). Data on clinicopathological characteristics and details about the surgeon were collected from a prospectively maintained database. A large tumour size was defined as tumour diameter equal or greater than $3 \mathrm{~cm}$ and a small tumour less than $3 \mathrm{~cm}$. A recurrence of first check cystoscopy was not necessarily a true recurrence at the previous site of resection but may have included new lesions in other sites of the bladder. All senior registrars began resecting bladder tumours under consultant supervision within 3 months of commencement and continued supervised training for at least 1-2 years.

SPSS software version 17.0 was used for statistical analysis and the results were expressed as frequency and percentages. Chi-square test was used to determine statistical significance. Ap of 0.05 or less was considered statistically significant.

\section{Results}

During the 10-year study period, 191 patients underwent TURBT with a diagnosis of NMIBC. However only 181 patients were relevant to the study (because SR experience of minimum 6 months was considered). Of which 155 (85.6\%) were male patients. The majority were pT1 tumours $(n=98$, $54.1 \%)$ and pTa were seen in $83(45.9 \%)$. None of the resected tumours had concomitant carcinoma in situ (CIS). Of 181 TURBT, 99 (54.7\%) were done by CUS and 82(45.3\%) were performed by the senior registrar with a training period of at least 6 months.

The overall DM positivity rate was $59.7 \%(n=108 / 181)$, for CUS: $63.6 \%(n=63 / 99)$ and SRs $54.9 \%(n=45 / 82)(p=0.232)$. Of all the pTa tumours ( $\mathrm{n}=83)$, DM positivity rate was $46.5 \%$ (20/43) for the CUS and 50\% (20/40) for SRs ( $p=0.751)$.
However, for pT1 cancers, DM positivity rates were $76.8 \%$ (43/56) for CUS and 59.5\%(25/42) for SR(p=0.067)(Table 1).

DM positivity rates were higher for females compared with males (pTa:50\% vs. $45.3 \%, \mathrm{p}=0.719 ; \mathrm{pT} 1:$ $78.6 \%$ vs. $70.7 \%$, $\mathrm{p}=0.651)$. For $\mathrm{pT} 1$ high grade tumours $(\mathrm{n}=52)$, the overall detrusor muscle positivity was $65.4 \%(n=34 / 52)$, for CUS: $75 \%(n=21 / 28)$ and Srs: 54.2\% $(n=13 / 24)(p=0.115)$ (Table 2). There was no significant association of size of tumour, sex or focality on presence of DM (Table 3 ).

Table 2: Presence of detrusor muscle in the TURBT specimen in pT1 high grade tumours

\begin{tabular}{|c|c|c|c|c|c|c|c|c|}
\hline & \multicolumn{3}{|c|}{ Small $(<3 \mathrm{~cm})$} & \multicolumn{3}{|c|}{ Large $(>3 \mathrm{~cm})$} & \multirow{3}{*}{$\begin{array}{c}\mathrm{P} \\
\text { value }\end{array}$} \\
\hline & & \multirow[t]{2}{*}{ Total } & \multicolumn{2}{|c|}{$\begin{array}{c}\text { DM } \\
\text { positive }\end{array}$} & \multirow[t]{2}{*}{ Total } & \multicolumn{2}{|c|}{$\begin{array}{c}\text { DM } \\
\text { positive }\end{array}$} & \\
\hline & & & $\mathrm{N}$ & $\%$ & & $\mathrm{~N}$ & $\%$ & \\
\hline \multicolumn{2}{|c|}{ Consultant } & 10 & 8 & 80.0 & 18 & 13 & 72.2 & 0.65 \\
\hline \multicolumn{2}{|c|}{ Four SRs } & 13 & 7 & 53.8 & 11 & 6 & 54.5 & 0.97 \\
\hline \multirow{4}{*}{$\begin{array}{c}\text { SR } \\
\text { Sub } \\
\text { division }\end{array}$} & SR-A & 4 & 2 & 50.0 & 4 & 0 & 0.0 & \multirow[t]{4}{*}{ - } \\
\hline & SR-B & 1 & 1 & 100 & 0 & 0 & 0.0 & \\
\hline & SR-C & 6 & 3 & 50.0 & 3 & 3 & 100 & \\
\hline & SR-D & 2 & 1 & 50.0 & 4 & 3 & 75.0 & \\
\hline
\end{tabular}

A significant number of patients were lost to follow-up at 3 months 36/181 (19.9\%) for first check cystoscopy. Recurrence rate at the first check cystoscopy was 41/181 $(22.7 \%)$ with no recurrence observed in 104/181 (57.5\%) patients (Table 2).

Analysis of 145/181 (80.1\%) followed up patients showed a positive recurrence at first check cystoscopy in $27.1 \%(23 / 85)$ and $30.0 \%(18 / 60)$ when detrusor muscle was present and absent in the first TURBT specimen respectively.

The correlation between early recurrence at the first check cystoscopy (at 3 months) and detrusor muscle status was analysed. We did not find a statistically significant difference (Odds ratio=0.87 (95\% Confidence Interval: 0.41-1.8, $\mathrm{p}=0.7)$. 
Table 3. Presence of detrusor muscle in the TURBT specimen in small and large bladder tumours dependent on sex and focality

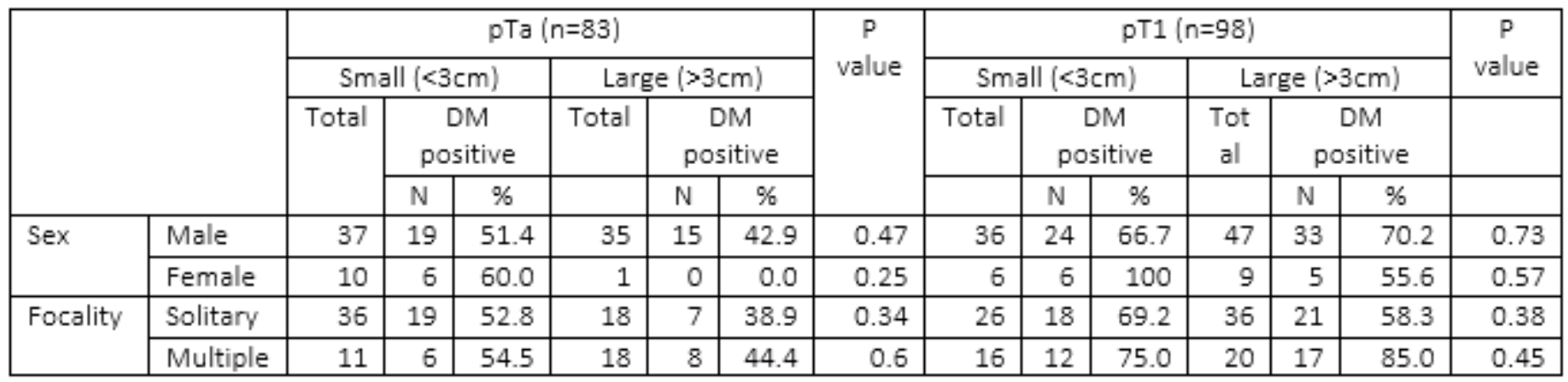

Table 4. Recurrence rate at the first check cystoscopy based on stage, presence/ absence of detrusor muscle for CUS and 4 SRs (collectively)

\begin{tabular}{|c|c|c|c|c|c|c|c|}
\hline & & & \multirow[t]{3}{*}{ Total } & \multicolumn{3}{|c|}{ Recurrence } & \multirow[t]{3}{*}{ P value } \\
\hline & & & & Yes & No & $\begin{array}{c}\text { Lost to follow } \\
\text { up }\end{array}$ & \\
\hline & & & & $\mathrm{N}(\%)$ & No (\%) & $\mathrm{N}(\%)$ & \\
\hline \multirow[t]{4}{*}{ Consultant } & \multirow[t]{2}{*}{$\mathrm{pTa}$} & DM present & 20 & $2(10.0)$ & $12(60.0)$ & $6(30.0)$ & \multirow[t]{2}{*}{0.6} \\
\hline & & DM absent & 23 & $4(17.4)$ & $15(65.2)$ & $4(17.4)$ & \\
\hline & \multirow[t]{2}{*}{ pT1 } & DM present & 43 & $12(27.9)$ & $18(41.9)$ & $13(30.2)$ & \multirow[t]{2}{*}{0.75} \\
\hline & & DM absent & 13 & $3(23.1)$ & $7(53.8)$ & $3(23.1)$ & \\
\hline \multirow[t]{4}{*}{ Four SRs } & \multirow[t]{2}{*}{ pTa } & DM present & 20 & $4(20.0)$ & $13(65.0)$ & $3(15.0)$ & \multirow[t]{2}{*}{0.73} \\
\hline & & DM absent & 20 & $6(30.0)$ & $12(60.0)$ & $2(10.0)$ & \\
\hline & \multirow[t]{2}{*}{ pT1 } & DM present & 25 & $5(20.0)$ & $19(76.0)$ & $1(4.0)$ & \multirow[t]{2}{*}{0.09} \\
\hline & & DM absent & 17 & $5(29.4)$ & $8(47.1)$ & $4(23.5)$ & \\
\hline
\end{tabular}

\section{Discussion}

TURBT is the essential surgical procedure used to diagnose, stage and treat primary NMIBC [7]. Ideally the initial TURBT should be thorough and complete. Many factors confound the adequacy of resection such as multiplicity, tumour location, surgeons' skills, quality of specimens and pathological analysis [8]. Herr analysed the results of a second TURBT 2-6 weeks after an initial TURBT in 150 consecutive patients evaluated for localised bladder tumours [7].

There was an initial incomplete TUR in $49 \%$ of stage pT1 tumours having no muscle submitted in the first TUR specimen compared with $14 \%$ when muscle was identified. The inclusion of DM in the first TURBT specimen is essential for accurate pathological staging for prognostication and further management, particularly when there is tumour in the lamina propria [9]. Dalbagni et al. showed that the DM was absent in $40 \%$ of pT 1 cancers [10].

In our study, DM in the TURBT specimen for pT1 tumours for the CUS and the four senior registrars were $76.8 \%$ and $59.5 \%$ respectively. In $75 \%$ of patients with pT 1 high grade tumours, DM was present in the TURBT specimen by CUS as opposed to $54.2 \%$ when performed by SRs. In contrast, it is well known that the DM status in the resected specimen has no prognostic significance in pTa tumours- those with no tumour infiltration of the lamina propria. In a multicentre retrospective study of 2451 patients with T1 HG (1990-2011) initially managed with BCG, 935 (38\%) were subjected to a re-TURBT [11]. DM was seen in the initial TURBT in 1768 $(72 \%)$.

Approximately $71 \%$ of 935 patients had residual tumour, with around $31 \%$ stage pT 1 and $40 \%$ stage pTa. Re-TURBT was favourable in terms of recurrence rate, progression, cancerspecific survival and overall survival only when the DM was absent at first TURBT. Therefore, they did not show a benefit of a re-TURBT after the initial TURBT that had DM in the pathological specimen [11]. Similarly, a study by Roupret el al showed a significantly lower rate of DM in junior surgeons compared with senior surgeons (74\% vs.61\%, $\mathrm{p}=0.02)$ [12]. Furthermore, recurrence at first check cystoscopy was low in the DM group (31\% vs. 53\%, p=0.01) [12].

In a 2-year study in Edinburgh in 241 patients of 356 (67.7\%), DM was present [13]. Urologists were grouped into seniors (urology trainees with 5-6 years of experience and 
consultants) and juniors (urological trainees of less than 5 years of experience). DM was present in $72.6 \%$ and $56.8 \%$ of TURBTs performed by senior and junior urologists respectively [13]. Jesuraj and colleagues described a significant surgeon-related variations in the presence of DM particularly in the higher grade cancers [14]. DM was present in around $46 \%$ in juniors as opposed to $67 \%$ among the seniors [15], with the highest risk being at the initial check cystoscopy done at 3 months after the initial TURBT [16].

In the present study, recurrence rate at the initial first check cystoscopy was $22.7 \%$ with no recurrences in $57.5 \%$. Nearly one fifth of patients were lost to follow-up at the first check cystoscopy.

In a European study of patients with NMIBC, Sylvester et al. demonstrated that the recurrence rate at initial check cystoscopy at 3 months was $12.1 \%$ with no recurrences in $79.7 \%$. Even in this series, $8.2 \%$ of 2,596 patients were lost to follow up at first check cystoscopy at 3 months [17].

In their study, $11 \%$ of NMIBC patients developed progression to muscle invasive bladder tumour. They showed the prognostic significance of the recurrence at the initial check cystoscopy on the subsequent progression to muscle invasive disease (at least T2). Progression was noted among 181 $(8.7 \%)$ of 2070 patients $(8.7 \%)$ with no recurrence at 3 months as opposed to $80(25.6 \%)$ of 313 patients with a recurrence at 3 months [17].

We did not analyse the progression in our study because of the increasingly higher lost to follow-up with subsequent check cystoscopies. We acknowledge some limitations of the present study. The first is the high rate of drop-outs after the initial TURBT of $19.9 \%$. Secondly, re-staging TUR at 2-6 weeks after initial TURBT was not analysed because it was seldom practised.

TURBT is the initial treatment of choice for NMIBC. Initial TURBT should be an oncologically sound operation (thorough and complete), one difficult to achieve. Detrusor muscle (DM) in the TURBT specimen is an essential prerequisite to define a complete resection.

This applies especially to pT1 bladder tumours with lamina propria infiltration. DM positivity rates for pT1 tumours resected by the experienced specialist and the four trainee surgeons were comparable to those reported in the world literature. However, we cannot be complacent about the high positive DM rates until these figures approach perfection while simultaneously maintaining patient safety (no bladder perforations).
All authors disclose no conflict of interest. The study was conducted in accordance with the ethical standards of the relevant institutional or national ethics committee and the Helsinki Declaration of 1975, as revised in 2000 .

\section{References}

1. Chavan S, Bray F, Lortet-Tieulent J, Goodman M, Jemal A. International variations in bladder cancer incidence and mortality. Eur Urol. 2014;66:59-73.

https://doi.org/10.1016/j.eururo.2013.10.001

2. Goonewardena S, De Silva W, De Silva M. Bladder cancer in Sri Lanka: experience from a tertiary referral center. International journal of urology. 2004;11:969-72.

https://doi.org/10.1111/j.1442-2042.2004.00930.x

3. Goonewardena SA, Jayarajah U, de Silva MC, Kuruppu SN, Fernando DH, Herath KB. Bladder cancer in women: a Sri Lankan study. Ceylon Medical Journal. 2019;64. https://doi.org/10.4038/cmj.v64i3.8952

4. Babjuk M, Böhle A, Burger M, Capoun O, Cohen D, Compérat $\mathrm{EM}$, et al. EAU guidelines on non-muscle-invasive urothelial carcinoma of the bladder: update 2016. Eur Urol. 2017;71:44761.https://doi.org/10.1016/j.eururo.2016.05.041

5. Jayarajah U, Herath K, Fernando M, Kuruppu S, Wickramanayaka U, Fernando I, et al. A clinico-pathological study of nonurothelial bladder cancers in a cohort of patients from a tertiary care urology unit in Sri Lanka. Ceylon Medical Journal. 2018;63. https://doi.org/10.4038/cmj.v63i2.8680

6. Furuse H, Ozono S. Transurethral resection of the bladder tumour (TURBT) for non-muscle invasive bladder cancer: Basic skills. Int J Urol. 2010;17:698-99.

https://doi.org/10.1111/j.1442-2042.2010.02556.x

7. Herr HW. The value of a second transurethral resection in evaluating patients with bladder tumors. The Journal of urology. 1999;162:74-76. https://doi.org/10.1097/00005392-199907000-00018

8. Herr HW, Donat SM. Quality control in transurethral resection of bladder tumours. BJU Int. 2008;102:1242-46. https://doi.org/10.1111/j.1464-410X.2008.07966.x

9. Goonewardena S, Jayarajah U, Kuruppu S, Herath H, Fernando D, Vickneswaran K. Clinical outcomes in a cohort of patients with $\mathrm{T} 1 \mathrm{high}$ grade urothelial bladder cancer not receiving intravesical bacillus Calmette-Guerin: a 15 year experience. Ceylon Medical Journal. 2018;63. https://doi.org/10.4038/cmj.v63i1.8626

10.Dalbagni G, Herr HW, Reuter VE. Impact of a second transurethral resection on the staging of $\mathrm{T} 1$ bladder cancer. Urology. 2002;60:822-24.

https://doi.org/10.1016/S0090-4295(02)01920-9

11. Gontero P, Sylvester R, Pisano F, Joniau S, Oderda M, Serretta V, et al. The impact of re-transurethral resection on clinical outcomes in a large multicentre cohort of patients with T1 high-grade/Grade 3 bladder cancer treated with bacille CalmetteGuérin. BJU international. 2016;118:44-52.

https://doi.org/10.1111/bju.13354 
12.Roupret M, Yates DR, Varinot J, Phe V, Chartier-Kastler E, Bitker $\mathrm{M}-\mathrm{O}$, et al. The presence of detrusor muscle in the pathological specimen after transurethral resection of primary pT1 bladder tumors and its relationship to operator experience. The Canadian journal of urology. 2012;19:6459-64.

13.Mariappan P, Zachou A, Grigor KM. Detrusor muscle in the first, apparently complete transurethral resection of bladder tumour specimen is a surrogate marker of resection quality, predicts risk of early recurrence, and is dependent on operator experience. Eur Urol. 2010;57:843-49.

https://doi.org/10.1016/j.eururo.2009.05.047

14. Jesuraj M, Harris M, Rogers A, Whiteway J. Completeness of first resection of bladder tumour depending on seniority of the surgeon. European Urology Supplements. 2008;7:138. https://doi.org/10.1016/S1569-9056(08)60267-5
15.Millan-Rodriguez F, Chechile-Toniolo G, Salvador-Bayarri J, Palou J, Vicente-Rodriguez J. Multivariate analysis of the prognostic factors of primary superficial bladder cancer. The Journal of urology. 2000;163:73-78. https://doi.org/10.1016/S0022-5347(05)67975-X

16.Mariappan P, Smith G, Lamb AD, Grigor KM, Tolley DA. Pattern of recurrence changes in noninvasive bladder tumors observed during 2 decades. The Journal of urology. 2007;177:867-75.

https://doi.org/10.1016/j.juro.2006.10.048

17.Sylvester RJ, van der Meijden AP, Oosterlinck W, Witjes JA, Bouffioux C, Denis L, et al. Predicting recurrence and progression in individual patients with stage Ta $\mathrm{T} 1$ bladder cancer using EORTC risk tables: a combined analysis of 2596 patients from seven EORTC trials. Eur Urol. 2006;49:466-77. https://doi.org/10.1016/j.eururo.2005.12.031 